\title{
A wireless communication system for energy and environmental monitoring
}

\author{
Dmytro Krush ${ }^{1}$, Christoph Cammin ${ }^{1}$, Ralf Heynicke ${ }^{1}$, Gerd Scholl ${ }^{1}$, and Bernd Kaercher ${ }^{2}$ \\ ${ }^{1}$ Electrical Measurement Engineering, Helmut Schmidt University, \\ University of the Federal Armed Forces Hamburg, Hamburg, Germany \\ ${ }^{2}$ Festo AG, CR-MC, Ruiter Strasse 82, 73734 Esslingen, Germany \\ Correspondence to: Dmytro Krush (dmytro.krush@hsu-hh.de)
}

Received: 12 August 2016 - Revised: 27 September 2016 - Accepted: 24 November 2016 - Published: 10 January 2017

\begin{abstract}
Custom-fit communication systems are key elements in modern cyber-physical sensor systems. Therefore a wireless communication system (WCS) for sensor/actuator communication has been developed to facilitate energy and environmental monitoring on the shop floor of industrial production sites. Initially, the distinct demands and requirements are described. As the WCS has been designed for new installations as well as for retrofitting already installed facilities, the WCS has to be able to coexist with other wireless communication systems already allocated in the same frequency band. The WCS handles measurement data from both, energy-autarkic sensors and fast line-powered sensors. Mobile users in the field equipped with mobile devices are served by the system, too. A modular hardware concept has been chosen for easy system modification or for the integration of new wireless standards. Finally, measured results for the coexistence capability are presented.
\end{abstract}

1

An ongoing tendency indicating increasing energy costs can be observed. Therefore energy efficiency of industrial manufacturing processes is becoming more important. Moreover, there is an additional demand by industrial manufacturers to assign carbon dioxide emissions to specific products and individual process steps.

The publicly funded research project ESIMA aims at measuring energy flows and at analyzing energy consumption in different production steps during the manufacturing process. The acronym "ESIMA" stands for "Optimierte Ressourceneffizienz in der Produktion durch Energieautarke Sensorik und Interaktion mit mobilen Anwendern" (English: "Optimized resource efficiency in the production process by energy-autarkic sensors and interaction with mobile users") (ESIMA, 2016). The ESIMA consortium consists of the following partners: FESTO AG (project coordinator) as manufacturer of pneumatic and electronic components, Varta Microbattery $\mathrm{GmbH}$ as manufacturer of storage battery systems, EnOcean $\mathrm{GmbH}$ as manufacturer of energy-autarkic sensors, the chair of Electrical Measurement Engineering at Helmut
Schmidt University, University of the Federal Armed Forces Hamburg, for the development of a robust wireless communication system, Hahn-Schickard as a service provider for sensor technology, c4c Engineering $\mathrm{GmbH}$ for software engineering, the Institute of Machine Tools and Production Technology at the TU Braunschweig for concept studies with respect to energy and resource efficiency, and Daimler AG as operator of the industrial target application.

One aspect in ESIMA is focused on interaction with mobile users. In the context of Industrie 4.0 a consistent information concept is realized, driven by the demands of the facility operators. Energy flows through the electrical and pneumatic supply lines of the production machinery are measured to extract and to visualize significant key figures. These key figures are used to reveal energy and cost savings. A role model has also been developed, including individual user groups like machine operators, maintainers, and management personal, which are provided with user-specific, especially reprocessed, and visually optimized data sets. Additionally, combined user groups, e.g., sitting in "shop-floor meetings", are taken into account. 
Table 1. Requirements for different wireless automation domains, extracted from VDI/VDE 2185 (2016).

\begin{tabular}{llll}
\hline Requirement & $\begin{array}{l}\text { Process } \\
\text { automation }\end{array}$ & $\begin{array}{l}\text { Building } \\
\text { automation }\end{array}$ & $\begin{array}{l}\text { Factory } \\
\text { automation }\end{array}$ \\
\hline Topology & $\begin{array}{l}\text { Ad-Hoc network; } \\
\text { radio/wire } \\
\text { combined }\end{array}$ & $\begin{array}{l}\text { Point-to- } \\
\text { point radio } \\
\text { network }\end{array}$ & $\begin{array}{l}\text { Ad-Hoc network; } \\
\text { radio/wire } \\
\text { combined }\end{array}$ \\
\hline Distance & $30 \mathrm{~m}->1000 \mathrm{~m}$ & $30 \mathrm{~m}-1000 \mathrm{~m}$ & $30 \mathrm{~m}-300 \mathrm{~m}$ \\
\hline Cycle time & $<1 \mathrm{~ms}-1 \mathrm{~s}$ & $>1 \mathrm{~s}$ & $\leq 1 \mathrm{~ms}$ \\
\hline User data & $30-1500$ bytes & $30-240$ bytes & $\leq 30$ \\
\hline
\end{tabular}

Besides electrical and pneumatic quantities, environmental quantities of the production sites like temperature and illumination intensity are measured with highly miniaturized embedded machine and application-specific sensor systems. In order to enable an easy and flexible integration into the production environment or to enhance existing production facilities, a wireless communication architecture is suggested. The wireless approach also has the advantage of providing measurement data and analysis results to mobile users and getting data from measurement points, where standard cabling is too expensive or too awkward to install.

\section{System requirements}

Sensor setup, configuration, and diagnosis as well as the accessibility and the processing of the sensor data are the key matters of concern in the ESIMA project. Mobility and flexibility requirements make a wireless communication system (WCS) necessary.

System requirements are derived from the needs of the individual user groups. In standard mode a measurement rate of $1 \mathrm{~Hz}$ has been chosen, with the option of increasing the sample rate to $10 \mathrm{~Hz}$ in ESIMA-streaming mode. For quantities like machine or room temperature with large time constants, the sample rate can be appropriately decreased. This ability is especially important for sensors, which have no energy harvesters, but which have to operate battery-powered over many months (Hornung et al., 2012). Thus, energy-autarkic sensors with long sleep times can also be integrated into the WCS.

The prospective number of sensors for the energy and environmental monitoring in ESIMA is on the order of 10 sensors per manufacturing cell with a footprint of $10 \mathrm{~m}$ by $10 \mathrm{~m}$. More sensors and also actuators are intended to be installed for enhanced functionalities, e.g., process monitoring. The following Table 1 is extracted from the guideline (VDI/VDE $2185,2016)$ itemizing typical system requirements in the various fields of automation and has also been taken as a guideline in ESIMA.

WLAN communication systems based on the standards according to IEEE $802.11 \mathrm{a} / \mathrm{b} / \mathrm{g} / \mathrm{n}$ exhibit RF bandwidths of at least $20 \mathrm{MHz}$ and gross data rates usually greater than $11 \mathrm{MBit} \mathrm{s}^{-1}$ (IEEE 802.11 WLAN, 2016). Thus they are often used for applications where high data throughputs are required, e.g., intra-logistic solutions. In comparison with Bluetooth or WirelessHART devices, which comply with the IEEE 802.15.1 and IEEE 802.15.4 standards, respectively, energy consumption is much higher, so that these devices cannot be used for energy-autonomous applications (Bluetooth SIG, 2016; HART Communication Foundation, 2016; IEEE 802.15.1 WPAN, 2016; IEEE 802.15.4, 2016; Scholl et al., 2013). Another drawback is that latency times for data transmission and jitter in latency times are not defined, so that this communication medium cannot be used for timecritical applications. Bluetooth was primarily designed for robust short-range applications offering sufficient data rates for many industrial applications and low energy consumption, but Bluetooth piconets are limited to seven active devices, also limiting the application spectrum (Bluetooth SIG, 2016; Weczerek and Pape, 2010). During the last few years WirelessHART has become the de facto standard for process automation applications, but cannot be used for applications, where cycle times on the order of several milliseconds and below must be realized (HART Communication Foundation, 2016; IEEE 802.15.4, 2016; Scholl et al., 2013). Thus the ESIMA network is the first system that can be used across the different application domains. WirelessHART and the ESIMA communication network also significantly differ from classical office communication standards as they offer all tools and resources, which are required for professional industrial sensor/actuator communication, i.e., appropriate engineering and development tools as well as tools for network setup, security management or network monitoring, and diagnosis or data acquisition. Thus WirelessHART and also ESIMA (yet to a smaller extent) offer substantially more than a bare data link like WLAN or Bluetooth and represent the connection to the cyber world for modern cyber-physical systems.

As many production environments are already equipped with wireless systems, in particular WLAN (IEEE 802.11 WLAN, 2016), and these systems are also utilized for production-critical data transmission such as, e.g., intraproduction site logistics, the established performance of these systems is protected by prohibiting any possible degradation. Thus, an additional WCS has to ensure a continuous coexistence with the existing wireless systems.

\section{Wireless communication technology}

An industrial environment is typically characterized by many metallic structures causing diffraction, multiple reflections, and shadowing, where there is often no line-of-sight between the transmitting and receiving antennas. Every radio channel can be characterized by its time-variant and frequencyselective behavior, where the coherence bandwidth is a char- 
acteristic quantity of a radio channel describing the frequency behavior (Rappaport, 2001). Many measurements of radio channels in industrial environments were performed and analyzed, resulting in the coherence bandwidth being on the order of a few $\mathrm{MHz}$ in the $2.4 \mathrm{GHz}$ industrial, scientific and medical (ISM) band (Wattar, 2010; Krueger et al., 2012). If the transmission bandwidth of a wireless system is significantly smaller than the coherence bandwidth of the radio channel, the radio channel can be treated as frequencyflat and the communication system is denoted as narrowband (Rappaport, 2001). This has the advantage that there is no need for complex radio channel equalization, as is the case for wide-band communication (Heynicke et al., 2008; Scholl et al., 2013; Kärcher et al., 2014). Accordingly, this reduction of the receiver's complexity is beneficial for low-power operation. More importantly, it has been shown that in complex industrial environments low-latency times in conjunction with a high reliability can be ensured using narrow-band wireless communication systems (Heynicke et al., 2008; Scholl et al., 2013; Kärcher et al., 2014). The implemented WCS is derived from the IEEE 802.15.1 standard (IEEE 802.15.1 WPAN, 2016) as an open standard and utilizes the $2.4 \mathrm{GHz}$ ISM band, as it is available in most countries. Another advantage is that there is a large availability of highly integrated commercial transceiver chips that can be tailored to the specific customer needs (Krush et al., 2016).

Gaussian frequency-shift keying (GFSK) is used as a modulation technique at a bit rate of $1 \mathrm{Mbits}^{-1}$. In accordance with the IEEE 802.15.1 standard (IEEE 802.15.1 WPAN, 2016), up to 81 channels are utilized with a channel spacing of $1 \mathrm{MHz}$. A so-called "blacklisting" is implemented to avoid frequencies already occupied by other wireless systems. The network has a star-shaped topology. The gateway provides the interface of the WCS to the wired LAN with the connected backend system or the database. Moreover, sensor nodes facilitate low-energy communication and a predefined latency time can be assured also for energy-autarkic sensors (Heynicke et al., 2008; Hornung et al., 2012).

The WCS provides bidirectional communication offering the possibility of reconfiguring sensors and returning acknowledge messages for correctly received packets to ensure a high safety level. As an additional advantage, wireless actuators can also be addressed.

\subsection{Protocol structure}

A combined time and frequency division multiple access scheme has been chosen. The sensors/actuators are assigned to one of up to four uplink tracks (ULs) for the uplink communication from the sensors/actuators to the gateway. The frequency spacing between the DL track and the UL tracks is at least $22 \mathrm{MHz}$. This frequency spacing allows one to operate a WLAN system between the DL track and the UL tracks. Additionally, crosstalk between the DL track and the UL

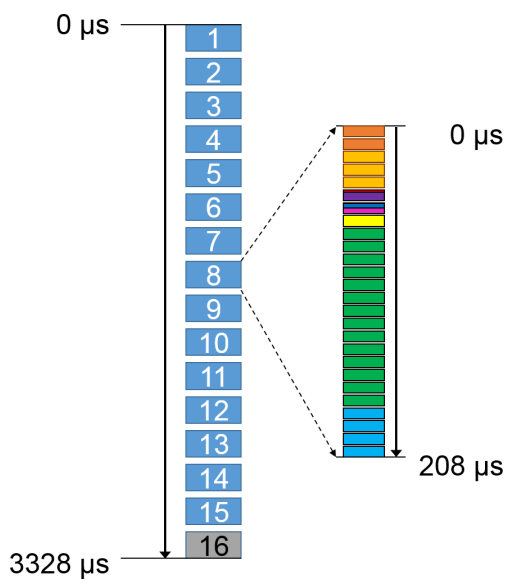

Figure 1. Downlink frame with 16 slots and a downlink packet.

tracks is sufficiently avoided. Measurements have shown that WLAN systems operated at frequencies close to the WCS do not degrade the WCS's performance, even if the power level of the WLAN is up to $20 \mathrm{~dB}$ higher than the power level of the WCS (Wattar, 2010).

Each UL track carries the communication load of up to 15 nodes, so that in total up to 60 sensor/actuator nodes can be served. There is only one downlink track (DL) for transmissions from the gateway to the sensor/actuator nodes. Data transmission is asymmetric due to the fact that in total more data have to be transmitted by the sensor/actuator nodes than data have to be received from the gateway.

The communication protocol is organized into time frames, where each frame has a duration of $3.328 \mathrm{~ms}$, so that in the case of disturbances two retransmits can be carried out within a time frame of $10 \mathrm{~ms}$. The structure of the frames is shown in Fig. 1. Each frame consists of 16 time slots, each lasting $208 \mu \mathrm{s}$. Within the 16th time slot at the end of each frame a frequency change is carried out. A frequencyhopping scheme is used to circumvent fading effects of the radio channel. A second advantage is the probability of interferences with other wireless systems being further reduced. Different frequency-hopping algorithms can be chosen, depending on the application, prospective environment, and specific requirements (Scholl et al., 2010; Krueger et al., 2012).

\subsection{Packet structure}

Each DL packet comprises a 16 bit preamble, a 24 bit sync word, and additional 24 bit for protocol management. The payload consists of 112 bit. A cyclic redundancy check (CRC) with a length of 32 bit is used for error detection, so the probability that a corrupted message will be falsely handled as an allowed message is below $10^{-9}$ (Hornung et al., 2012). The structure of the DL packet is presented in Fig. 2. The structure of a UL packet is shown in Fig. 3. The UL 


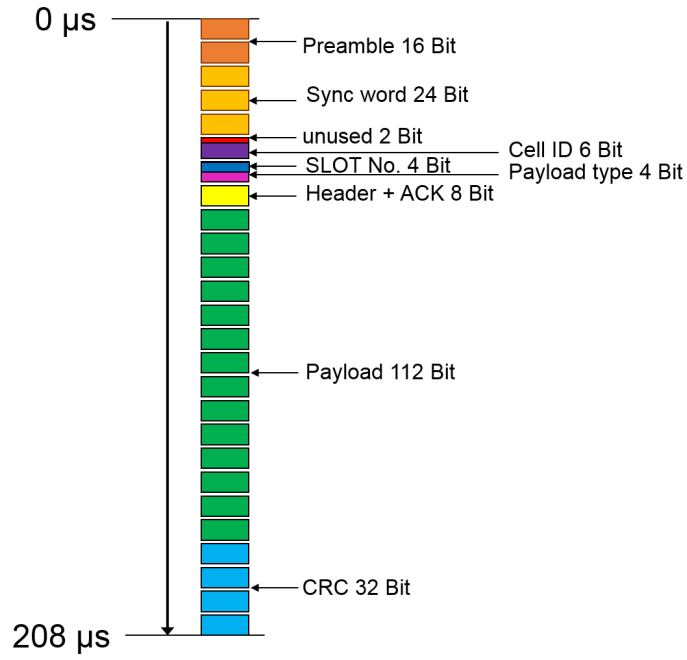

Figure 2. Downlink packet.

packet contains an additional guard interval of $8 \mu$ s, ensuring that multiple echoes caused by the radio channel will have decayed and that the gateway's receivers can cope with the fast packet handling, even if the sensor/actuator nodes are imperfectly synchronized. As 2 bytes for the protocol management are sufficient, the total duration of a UL packet is $208 \mu$ s too.

\subsection{Additional features}

Beyond the scope of energy monitoring, the capabilities described below were also implemented to cover additional prospective applications.

\subsubsection{Time stamping}

Due to the relatively low sample rate of typically $1 \mathrm{~s}$ and the fast processing time of the data wirelessly sent by the sensor/actuator nodes, it is sufficient that the individual information messages are time-stamped when they arrive at the interface of the backend database. This omits the need for data synchronization of the sensor/actuator nodes, resulting in less computational effort and hence a reduced energy consumption. Also, data size and thus channel occupancy can be kept small.

\subsubsection{Streaming mode}

To allow a higher time resolution of sensor data, a streaming mode has been implemented, which has to be initiated by the user via the backend system.

After the streaming mode has been triggered, a control command will be sent to the gateway by the backend system. When the sensors receive the control signal in the downlink message, they increase their wireless transmission rate. Supplementary to Sect. 2, the transmission rate can then be

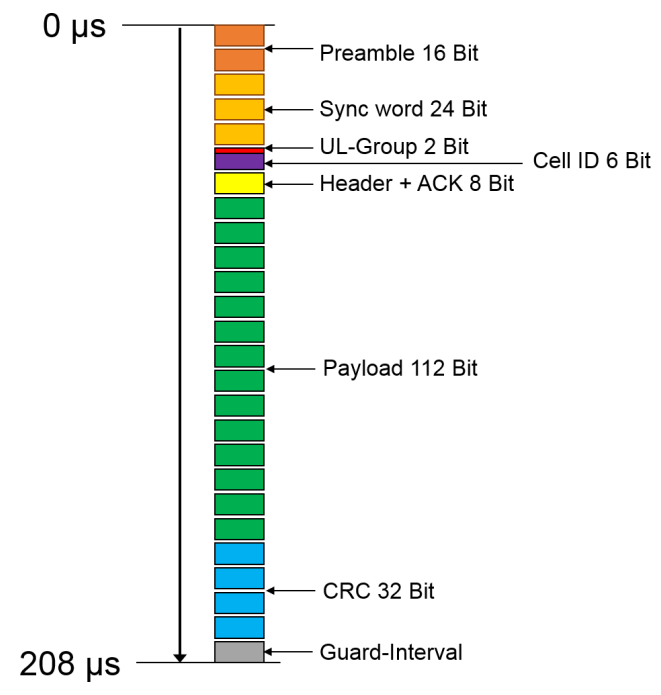

Figure 3. Uplink packet.

increased beyond 10 up to $100 \mathrm{~Hz}$. The net-data rate equals $11.2 \mathrm{kbit}$ per sensor/actuator node in the uplink. Assuming measurement values with 8 bit resolution, sample rates of up to $1.4 \mathrm{kHz}$ for each node can be realized if multiple measurement values are transmitted in one packet.

\section{Hardware architecture}

In what follows the hardware components of the gateway and the sensor nodes are described.

\subsection{Hardware of the gateway}

The gateway is realized energy-efficiently and compactly on the basis of a Xilinx Zynq system-on-chip (SoC), combining a field-programmable gate array (FPGA) with an ARM dualcore microcontroller (Xilinx, Inc., 2016). The structure of the hardware architecture is shown in Fig. 4.

VHDL state machines were implemented in the logic section of the SoC to control the radio transceivers. A dual-port RAM is employed as a buffer for data communication between the ARM microcontroller and the VHDL structures. Data and protocol handling is organized with the help of the ARM microcontroller, which also transfers data between a LAN interface and the VHDL structures.

Figure 5 shows a photo of a gateway demonstrator. That gateway is equipped with five radio transceiver modules, where one is based on a Texas Instruments/Chipcon CC2400 transceiver chip (Texas Instruments, Inc., 2016) to transmit DL packets due to the ability for fast frequency change and their spectral performance in transmit mode. Four radio transceiver Nordic NRF 2401A transceiver chips (Nordic Seminconductor, 2016) have been chosen in the uplink part of the gateway due to their selectivity in receive mode and their ability for fast frequency change, too. It is possible to 


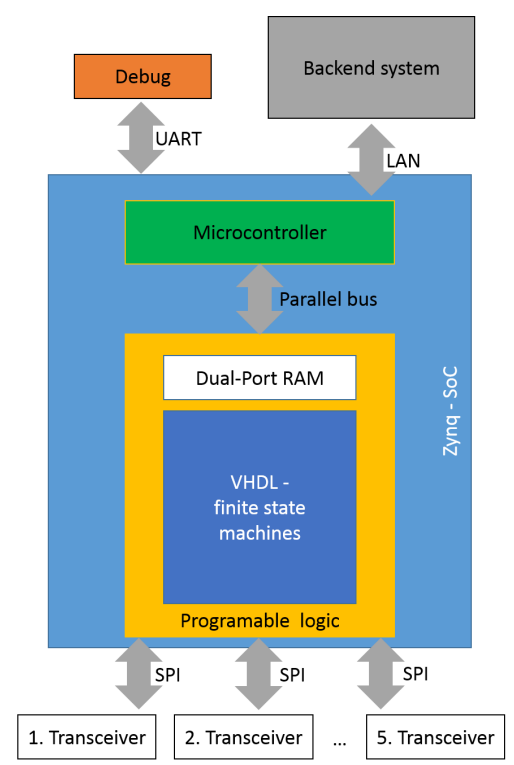

Figure 4. Hardware architecture of the gateway.

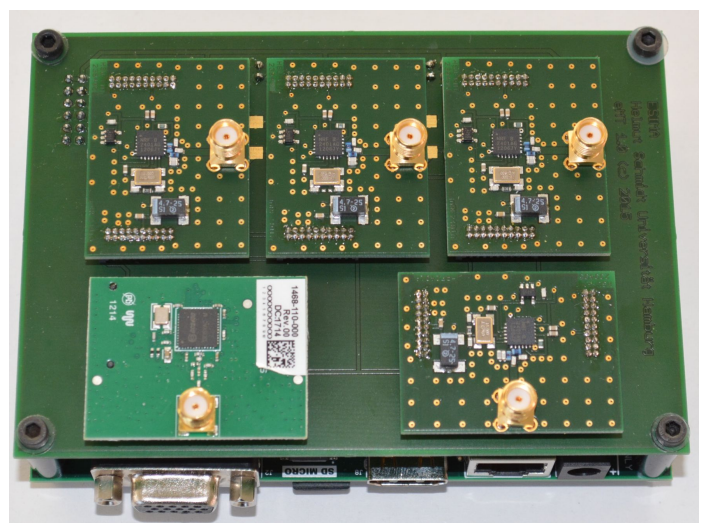

Figure 5. Photo of the demonstrator gateway.

modify the gateway easily owing to its modular design. As an example, the radio transceivers above can be replaced, e.g., by EnOcean transceivers (EnOcean, 2016). A photo of the gateway inside a housing is shown in Fig. 6. Four antennas for the UL tracks are integrated into the box on a common ground plane. The transmit signal for the DL signal is transferred to an external antenna. The housing was 3-D-printed for compactness.

\subsection{Hardware of the sensors/actuators}

The generic sensor/actuator architecture is shown in Fig. 7. To ensure a quick and comfortable exchange of components, specific connectors were standardized and employed. The internal communication between the main controller and the transceiver is realized via SPI. Radio transceiver modules based on the Texas Instruments CC2541 SoC (Texas Instruments, 2016) are used as they already include a microcon-

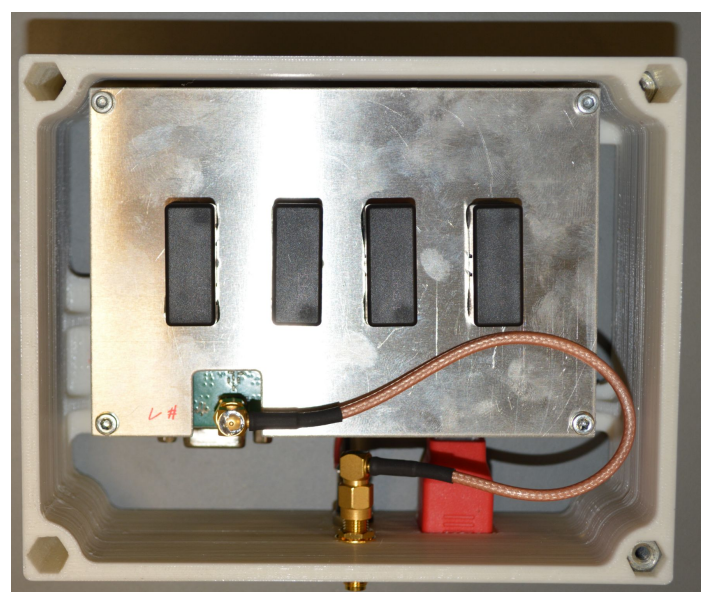

Figure 6. Photo of the gateway with internal antennas inside a housing.

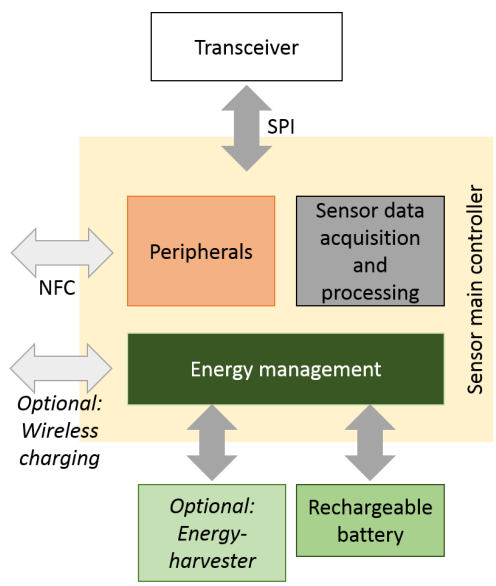

Figure 7. Generic hardware architecture of the sensors/actuators.

troller to handle the radio control and media access. Nearfield communication (NFC) is used for sensor/actuator configuration prior to installation and for additional service functionalities. Energy management software is implemented in the main controller, allowing one to reduce the communication load or even to interrupt communication in a defined way if energy is low.

All sensors are equipped with a rechargeable battery. Even the line-powered sensors are battery buffered to facilitate condition monitoring of the power supply line itself. Some sensor types are equipped with an energy harvester or with electronic modules for wireless charging. In the following Table 2, the sensor devices, developed by the ESIMA partners up to now, are listed.

Two different sensor designs for compressed air were implemented for different applications: the MS6-type sensors are intended for fixed installation. They contain a turbinebased generator unit driven by compressed air to charge the internal battery. The measuring range is $0-10$ bar pressure. 
Table 2. Sensor types in ESIMA.

\begin{tabular}{llll}
\hline Name & Domain & Included sensors & $\begin{array}{l}\text { Power } \\
\text { supply }\end{array}$ \\
\hline $\begin{array}{l}\text { pQ-Sensor, } \\
\text { type: MS6 }\end{array}$ & $\begin{array}{l}\text { Compressed } \\
\text { air }\end{array}$ & $\begin{array}{l}\text { Pressure, } \\
\text { flow }\end{array}$ & Generator \\
\hline $\begin{array}{l}\text { pQ-Sensor, } \\
\text { type: Ad-Hoc }\end{array}$ & $\begin{array}{l}\text { Compressed } \\
\text { air }\end{array}$ & $\begin{array}{l}\text { Pressure, } \\
\text { flow }\end{array}$ & $\begin{array}{l}\text { Wireless } \\
\text { charging }\end{array}$ \\
\hline E-Meter & Electrics & $\begin{array}{l}\text { Voltage, current, } \\
\text { frequency (three-phase) }\end{array}$ & $\begin{array}{l}\text { Line- } \\
\text { powered }\end{array}$ \\
\hline EIS sensor & $\begin{array}{l}\text { Environment } \\
\text { (ambience) }\end{array}$ & $\begin{array}{l}\text { Air flow (2-D), } \\
\text { air pressure, temperature, } \\
\text { humidity, } \mathrm{CO}_{2}, \text { luminosity }\end{array}$ & $\begin{array}{l}\text { Line- } \\
\text { powered }\end{array}$ \\
\hline
\end{tabular}

By using one of three different internal bypasses, the measuring range for compressed air flow rate can be chosen between 2-200, 10-1000, or 50-5000 $\mathrm{L} \mathrm{min}^{-1}$. The Ad-Hoc type sensor is intended for flexible temporary mobile use. It has measuring ranges of $0-10$ bar pressure and $2-200 \mathrm{~L} \mathrm{~min}^{-1}$ flow rate. To keep the housing lightweight and compact, the internal generator unit was omitted. The internal battery lasts for $10 \mathrm{~h}$ continuous operation with a measuring and data transmission rate of $1 \mathrm{~Hz}$ each.

The E-Meter measures voltages, currents, phase angles $(\cos (\phi))$, and frequencies such that all relevant parameters for a three-phase power network as active, reactive, and apparent power can be calculated and transmitted. By selecting different current clamps, measurements can be taken over a wide range.

The EIS (Environmental Information System) environmental sensor is intended to measure quantities related to environmental conditions around and within the machines and for working safety and comfort. Measured quantities are temperature, humidity, pressure, flow, and $\mathrm{CO}_{2}$ content of the surrounding air and luminosity. Trends and forecasts for the quantities related to the surrounding air can be derived by measuring the air flow rate horizontally in two dimensions at several installed EIS sensors. Estimations can also be derived for, e.g., the heating control system of the production hall. Another example is the measurement of the luminosity to determine whether there is enough light in the various working areas.

\section{Coexistence}

The coexistence behavior of the WCS is not only based on blacklisting, but also on frequency-hopping schemes especially developed for operation in industrial environments. In the next paragraph, characteristics of blacklisting algorithms are presented and in the second part a measurement of a coexistence test is presented.

\subsection{Blacklisting}

Blacklisting denotes a frequency or channel separation technique to manually or automatically separate different wireless systems operations in the same frequency band. It can be applied in general, but some limitations have to be taken into account: from a regulatory perspective there has to be a minimum number of hopping frequencies for such frequencyhopping devices that are used in the WCS. According to EN 300328 at least 15 frequency channels have to be employed by the devices in this case (ETSI, 2016).

WLAN channels in the $2.4 \mathrm{GHz}$ band usually show a bandwidth of $20 \mathrm{MHz}$ (e.g., 802.11n) or $22 \mathrm{MHz}$ (e.g., 802.11b) (IEEE 802.11 WLAN, 2016). Thus, if three WLAN channels are allocated, there remain 15 or 21 frequency channels for the operation of the WCS, respectively. As described in Sect. 3, one fully employed WCS with 60 sensor/actuator nodes in four UL tracks needs in total five $1 \mathrm{MHz}$ wide channels at a time for no loss of performance or reduction of sensor nodes.

The frequency-hopping schemes are designed to operate at the maximum three fully employed WCSs at one place with minimal overlapping of the frequency channels, so the WCSs do not have to be synchronized with each other. Therefore it is possible to operate three fully employed WCSs in parallel with three WLAN systems, if sufficient filtering and spectral masks are preconditioned. Therefore no constraints regarding the number of nodes or the timing behavior exist.

\subsection{Coexistence test}

A coexistence scenario, which can often be found in the target applications, is that three WLAN systems are already operated in the $2.4 \mathrm{GHz}$ ISM band. Typically, channels 1,7 , and 13 are chosen, occupying the frequency bands between 2402 and 2422, 2432 and 2452, and 2462 and $2482 \mathrm{MHz}$ (IEEE 802.11 WLAN, 2016). These bands are cancelled ("blacklisted") in the frequency list for possible data transmissions. The test setup for this scenario is presented in Fig. 8. The WLAN access points are connected via $30 \mathrm{~dB}$ attenuators to a 16-port power combiner/splitter. The downlink channel is attenuated by $20 \mathrm{~dB}$. For the sensor nodes $10 \mathrm{~dB}$ attenuators are used. The isolation between the ports is $30 \mathrm{~dB}$; the total insertion loss is $12.5 \mathrm{~dB}$, typically (Mini-Circuits, 2016). The power levels at the different receivers given in Table 3 are calculated by typically applying $3.5 \mathrm{~dB}$ additional loss per connection due to cables, adapters, and connectors. The attenuations are chosen to have power levels comparable with the target application.

With the help of a Tektronix real-time spectrum analyzer (Tektronix, 2016), signals can be monitored precisely. A typical spectrogram is shown in Fig. 9. The long narrow tracks between the WLAN packets indicate the downlink packages, whereas the short packages represent uplink data. The lengthy transmissions in Fig. 9 of the WCS demonstrate that 
Table 3. Receive power levels in $\mathrm{dBm}$.

\begin{tabular}{crrrr}
\hline \multirow{2}{*}{ Power $(\mathrm{dBm})$} & \multicolumn{4}{c}{ Transmitter } \\
\cline { 2 - 5 } & Gateway, DL & Sensor & WLAN \\
\hline \multicolumn{2}{c}{ Output power $(\mathrm{dBm})$} & 0 & 0 & +10 \\
\hline \multirow{4}{*}{ Receiver } & Gateway, UL & -66 & -56 & -56 \\
& Sensor & -76 & -66 & -66 \\
& WLAN & -96 & -86 & -111 \\
& RSA & -66 & -56 & -66 \\
\hline
\end{tabular}

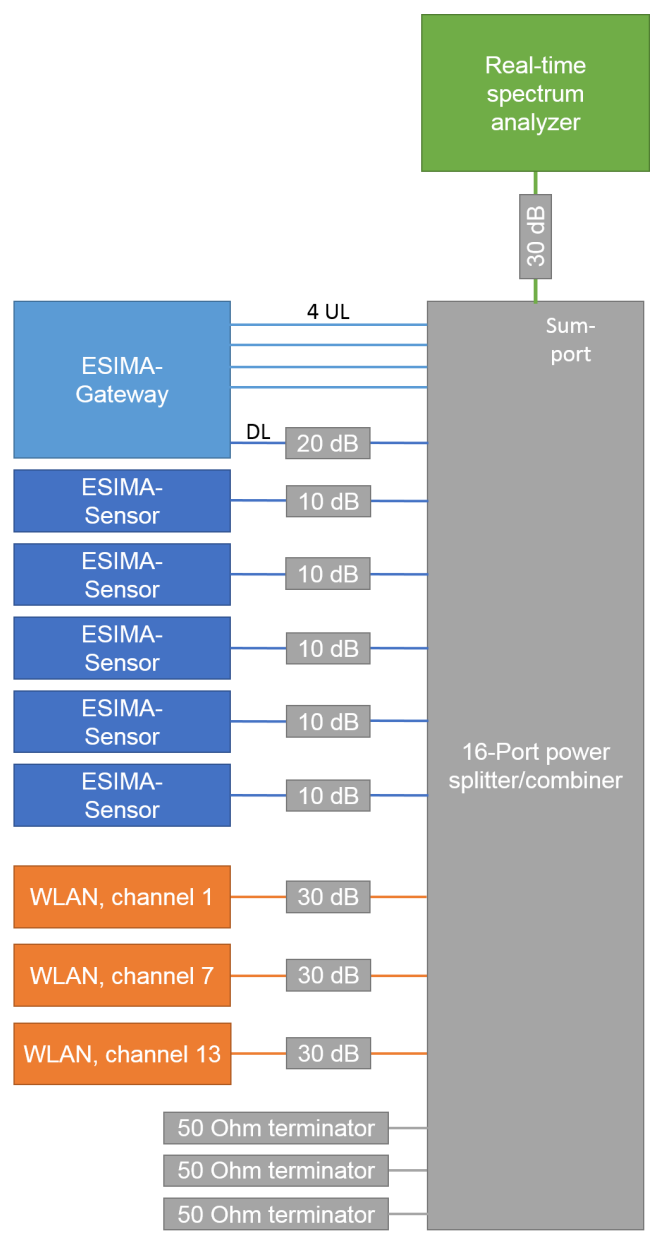

Figure 8. Test setup for coexistence investigations.

the downlink packets are sent for $3.328 \mathrm{~ms}$ without gaps. For this setup five sensors have been used. Three sensors transmit in every second frame, whereas the remaining two sensors transmit in the alternating frames, respectively. The frequency spacing between the UL tracks varies in time according to the hopping algorithm. No interference between the WCS and the WLAN systems could have been measured in this laboratory test setup. An additional detailed study on the coexistence behavior of the WCS is presented in Cammin et al. (2016).

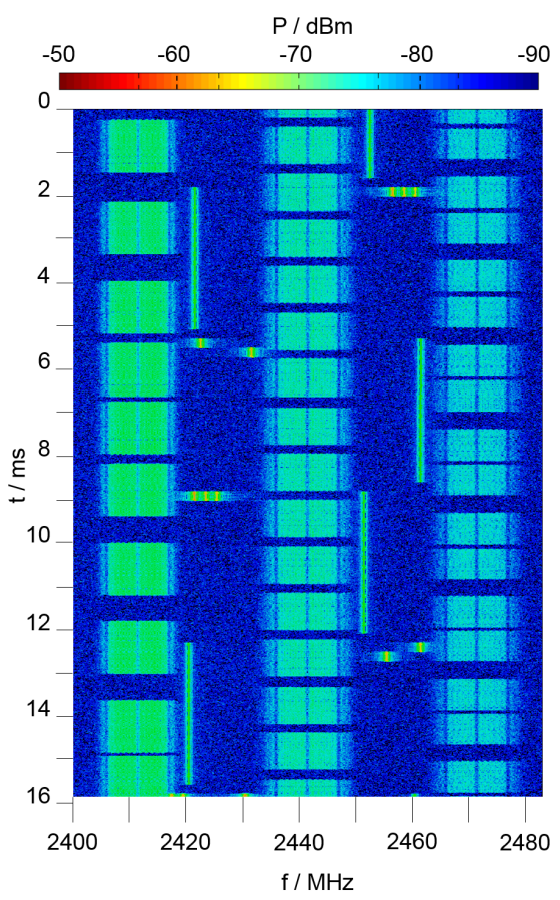

Figure 9. Spectrogram of the test setup.

\section{Conclusions}

A new wireless communication system (WCS) to facilitate energy monitoring for resource-efficient production was presented, enabling end users to precisely monitor energy flows and to develop energy-saving strategies in the field. The WCS provides customized energy-autarkic sensors as well as continuously powered sensors, offering higher data rates. Utilizing a blacklisting and application-specific frequencyhopping algorithms, the WCS is able to efficiently use the available spectrum together with the other wireless systems around. A defined maximum latency time below $10 \mathrm{~ms}$ can be achieved at a reliability level comparable with wired sensor/actuator networks. Thus, the requirements of energy and environmental monitoring in industrial applications are entirely fulfilled.

Acknowledgements. This work was done in research project ESIMA, which is funded by the Federal Ministry for Education and Research (BMBF) and managed by the VDI/VDE-IT. The authors would like to thank the project partners for their support and cooperation.

Edited by: J. Auge

Reviewed by: two anonymous referees 


\section{References}

Bluetooth SIG: Bluetooth Core Specification 4.2, available at: https: //www.bluetooth.com/specifications/adopted-specifications, last access: 23 June 2016.

Cammin, C., Schulze, C., Krush, D., Heynicke, R., Scholl, G., Thiede, S., and Hermann, C.: Coexisting Wireless Sensor Networks in Cyber-Physical Production Systems, in: 2016 IEEE International Conference on Emerging Technologies and Factory Automation, 1-4, doi:10.1109/ETFA.2016.7733593, 2016.

EnOcean: Batterielose Funktechnik von EnOcean für wartungsfreie Sensoren, available at: https://www.enocean.com/de/, last access: 23 June 2016.

ESIMA: Optimierte Ressourceneffizienz in der Produktion durch energieautarke Sensorik und Interaktion mit mobilen Anwendern, available at: http://esima-projekt.de/, last access: 23 June 2016.

ETSI: EN 300328 V1.9.1 (2015-02) Electromagnetic compatibility and Radio spectrum Matters (ERM); Wideband transmission systems; Data transmission equipment operating in the $2,4 \mathrm{GHz}$ ISM band and using wide band modulation techniques, available at: http://www.etsi.org/deliver/etsi_en/300300_ 300399/300328/01.09.01_60/en_300328v010901p.pdf, last access: 30 August 2016.

HART Communication Foundation: WirelessHART, available at: http://de.hartcomm.org/main_article/wirelesshart.html, last access: 30 August 2016.

Heynicke, R., Kruger, D., Wattar, H., and Scholl, G.: Modular wireless fieldbus gateway for fast and reliable sensor/actuator communication, in: 2008 IEEE International Conference on Emerging Technologies and Factory Automation, 1173-1176, doi:10.1109/ETFA.2008.4638548, 2008.

Hornung, R., Heynicke, R., and Scholl, G.: Schnelles drahtloses Funknetzwerk mit energieautarken Kommunikationsknoten, 6.1, 589-597, doi:10.5162/sensoren2012/6.1.1, 2012.

IEEE 802.11 WLAN: IEEE-SA-IEEE Get 802 Program - 802.11: Wireless LANs, available at: http://standards.ieee.org/about/get/ 802/802.11.html, last access: 4 July 2016.

IEEE 802.15.1 WPAN: IEEE SA - 802.15.1-2005 - IEEE Standard for Information technology - Local and metropolitan area networks- Specific requirements - Part 15.1a: Wireless Medium Access Control (MAC) and Physical Layer (PHY) specifications for Wireless Personal Area Networks (WPAN), available at: https://standards.ieee.org/findstds/standard/802.15. 1-2005.html, last access: 23 June 2016.

IEEE 802.15.4: IEEE SA - 802.15.4-2015 - IEEE Standard for Low-Rate Wireless Networks, available at: https://standards.ieee. org/findstds/standard/802.15.4-2015.html, last access: 23 June 2016.

Kärcher, B., Heynicke, R., and Scholl, G.: WSAN - Wireless - Kommunikation auf der Sensor/Aktorebene auf dem Weg von der Spezifikation zur Umsetzung, in: KommA 2014 - 5. Jahreskolloquium Kommunikation in der Automation, edited by: Jasperneite, J. and Jumar, U., http://www.jk-komma.de/images/ pdf/fullpaper/paper13.pdf, last access: 30 August 2016, 2014.
Krueger, D., Heynicke, R., and Scholl, G.: Wireless sensor/actuatornetwork with improved coexistence performance for $2.45 \mathrm{GHz}$ ISM-band operation, in: 2012 9th International MultiConference on Systems, Signals and Devices (SSD), 1-5, doi:10.1109/SSD.2012.6198099, 2012.

Krush, D., Cammin, C., Heynicke, R., and Scholl, G.: Standardisierung eines schnellen drahtlosen Sensor/Aktor-Netzwerkes fuer die Fertigungsautomatisierung, tm - Technisches Messen, 83, 201-207, doi:10.1515/teme-2015-0118, available at: http://www.degruyter.com/view/j/teme.2016.83.issue-4/

teme-2015-0118/teme-2015-0118.xml, last access: 23 June 2016.

Mini-Circuits: Coaxial Power Splitter/Combiner ZC16PD-2185, available at: https://www.minicircuits.com/pdfs/ZC16PD-2185. pdf, last access: 23 June 2016.

Nordic Seminconductor: nRF2401A/2.4 GHz RF/Home - Ultra Low Power Wireless Solutions from NORDIC SEMICONDUCTOR, available at: https://www.nordicsemi.com/chi/node_176/2. 4GHz-RF/nRF2401A, last access: 23 June 2016.

Rappaport, T. S.: Wireless Communications: Principles and Practice, Prentice Hall, Upper Saddle River, NJ, 2nd edn., 2001.

Scholl, G., Heynicke, R., and Krueger, D.: Steigerung der Robustheit drahtloser Sensor/Aktor - Feldbussysteme durch prozessorientiertes adaptives Frequency-Hopping, in: Wireless Technologies - 12. Kongress 22-23 September 2010, edited by: Wollert, J. F., VDI Verlag, Düsseldorf, 87-96, doi:10.13140/2.1.5119.5529, 2010.

Scholl, G., Heynicke, R., Krueger, D., and Hornung, R.: Wireless Automation, C3, 379-383, doi:10.5162/sensor2013/C3.1, 2013.

Tektronix: RSA6000 Spectrum Analyzer - Tektronix, available at: http://www.tek.com/spectrum-analyzer/rsa6000, last access: 4 July 2016.

Texas Instruments: CC2541 - Bluetooth/Bluetooth Low Energy Wireless Connectivity - Description \& parametrics, available at: http://www.ti.com/product/CC2541, last access: 4 July 2016.

Texas Instruments, Inc.: CC2400 - Proprietary $2.4 \mathrm{GHz}$ - Wireless Connectivity - Description \& parametrics, available at: http: //www.ti.com/product/CC2400, last access: 23 June 2016.

VDI/VDE 2185: VDI/VDE 2185 Blatt 1:2007-09 Radio based communication in industrial automation, available at: http://www. beuth.de/de/technische-regel/vdi-vde-2185-blatt-1/101776911, last access: 30 August 2016.

Wattar, H.: Robuste echtzeitfähige Funkkommunikation in der Fertigungsautomatisierung, Ph.D. thesis, Helmut-SchmidtUniversität, 2010.

Weczerek, J. and Pape, A.: Koexistenz von Bluetooth und WLAN in der industriellen Praxis, in: Wireless Technologies - 12. Kongress 22-23 September 2010, edited by: Wollert, J. F., VDI Verlag, Düsseldorf, 119-126, 2010.

Xilinx, Inc.: Zynq-7000 All Programmable SoC, available at: http:// www.xilinx.com/products/silicon-devices/soc/zynq-7000.html, last access: 23 June 2016. 